\title{
Can diplopia complaint be reduced by telerehabilitation in multiple sclerosis patient during the pandemic?: A case report
}

\author{
Esra Dogru-Huzmeli ${ }^{1}$ (D) Taskin Duman $^{2} \cdot$ Ayse Idil Cakmak $^{3} \cdot$ Ufuk Aksay $^{1}$
}

Received: 5 March 2021 / Accepted: 16 March 2021 / Published online: 24 March 2021

(C) Fondazione Società Italiana di Neurologia 2021

\begin{abstract}
Hospital visits and regular rehabilitation of chronic patients due to COVID-19 pose a risk. Therefore, patients with chronic illnesses who need regular rehabilitation have been victims of the pandemic process. Because of their fear of being infected, they were deprived of the chance of their symptoms being rehabilitated. Therefore, it is extremely important to rehabilitate individuals with chronic illnesses in need of rehabilitation through telerehabilitation. In this study, we aimed to show the effect of CawthorneCooksey exercises to be applied through telerehabilitation on eye movements, vision, and quality of life in a patient suffering from diplopia due to multiple sclerosis (MS). It has been found that Cawthorne-Cooksey exercises improve the quality of life and reduce the complaints of diplopia in MS patients with diplopia. In addition, the patient verbally stated that his balance increased after Cawthorne-Cooksey exercises. As a result, Cawthorne-Cooksey exercises are a rehabilitation method that gives positive results in the treatment of diplopia and it is recommended to apply this method via telerehabilitation.
\end{abstract}

Keywords Diplopia $\cdot$ Telerehabilitation $\cdot$ Multiple sclerosis

Multiple sclerosis, which is an inflammatory, demyelinating, and neurodegenerative pathology, can cause many eye disorders. Optic neuritis cases with optic nerve inflammation can be seen in approximately $20 \%$ of MS patients. Some reports have shown that $1 / 3$ of MS patients live visual symptoms. MS can significantly reduce the living standards of people due to vision-related diseases. The most common symptoms are low vision, decreased contrast sensitivity, discomfort in binocular vision, visual field abnormalities, decreased color vision, blurred vision, and double vision (diplopia). It is known that visual complaints negatively affect the balance and consequently prevent the person from performing daily living

Esra Dogru-Huzmeli

esradogru001@hotmail.com

1 Physiotherapy and Rehabilitation Department, Health Science Faculty, Hatay Mustafa Kemal University, Hatay, Turkey

2 Department of Neurology, Tayfur Ata Sokmen Medicine Faculty, Hatay Mustafa Kemal University, Hatay, Turkey

3 Department of Ophtalmology, Tayfur Ata Sokmen Medicine Faculty, Hatay Mustafa Kemal University, Hatay, Turkey activities. Limitations in daily living activities also cause the quality of life of people to deteriorate [1-5].

In the 1940s, British otolaryngolog Terence Cawthorne observed that some patients showed faster recovery with sequential and rapid head movements, and developed the eye rehabilitation protocol Cawthorne and Cooksey together with physiotherapist Cooksey. The exercises that are used today, based on the Cawthorne-Cooksey exercises, help the rehabilitation of the patients by supporting their movement after vestibular lesions. The Cawthorne-Cooksey protocol is based on the concepts of habituation and sensor substitution (displacement).

Diplopia seen in our patient with MS was directed to us by the neurologist for rehabilitation during the COVID-19 process because diplopia extremely disturbed the patient. Since the period when the patient was referred to us coincided with the pandemic process and the patient had a chronic illness and we did not want to put him at risk of being infected, we decided to rehabilitate our patient via the telerehabilitation method. When our patient agreed with the telerehabilitation, we applied telerehabilitation for 30 sessions to our patient.

Hospital visits and regular rehabilitation of chronic patients due to COVID-19 pose a risk. Therefore, patients with chronic illnesses who need regular rehabilitation have been victims of the pandemic process. Because of their fear of being infected, 
they were deprived of the chance of their symptoms being rehabilitated. Therefore, it is extremely important to rehabilitate individuals with chronic illnesses in need of rehabilitation via telerehabilitation.

Telerehabilitation (TR) is based on providing patients with remote access to rehabilitation services using communication technology. Today, by using TR services in the evaluation and treatment of many diseases, patients and the rehabilitation team save time, labor and treatment costs. TR, which is delivered through advanced technologies by adopting various application models, continues to develop day by day in parallel with communication technology [1-5].

In this study, we aimed to show the effect of CawthorneCooksey exercises to be applied via telerehabilitation on eye movements, vision and quality of life in a patient suffering from diplopia due to MS.

\section{Case}

The study was carried out in Hatay Mustafa Kemal University Faculty of Health Sciences, Department of Physiotherapy and Rehabilitation. One patient with a diagnosis of MS and diplopia was included in the study.

The case was 39 years old. His MS type was relapsingremitting and his disease duration was 15 years. His Expanded Disability Status Scale score was 1. He was married and working as a teacher.

Cawthorne-Cooksey exercises were applied to the patient participating in the study through telerehabilitation for 30 sessions, 3 sessions in a week. WhatsApp video chat was used for the connection. The same evaluations made before the application were repeated after the application. Firstly, the patient's demographic information and anamnesis were obtained and Short Form-36 (SF-36) scale was applied to evaluate the quality of life. It is accepted that the higher score on the SF-36 scale, the higher quality of life [6].
It is seen that there were an obvious increase and improvement in all parameters of the patient's quality of life (Table 1). The patient also expressed this verbally himself.

\section{Eye examination findings}

The patient, who had a complaint of double vision for 10 years, started to have low vision in both eyes in 2007. When the patient applied to the ophthalmology clinic, he stated that he had an MS attack in the form of visual impairment 3 times in 10 years. The patient was evaluated in the ophthalmology clinic in pretelerehabilitation and post-telerehabilitation terms.

According to the results of the ophthalmologic examination performed in the pre-telerehabilitation term, it was determined that direct and indirect light reflexes were bilaterally positive, and relative afferent pupillary infection was absent. The patient's color vision test (ishihara test) was found to be bilaterally within normal limits. It was observed that the patient had a slight head position and this position was slightly tilted to the right. When the alignment of the eyes was examined, it was determined that the eyes were ortophoric in the primary position and the eyes came slightly from outside in the openingclosing test.

When eye movements were evaluated, the right eye outward movement $(-2)$ degree was limited; the left eye was found to be restricted to $(-4)$ degrees. The patient stated that there was no pain during eye movements, but diplopia increased especially when looking to the right.

Intraocular pressure was measured with a pneumometer as $14 \mathrm{mmHg}$ on the right and $13 \mathrm{mmHg}$ on the left. Bestcorrected visual acuity was $-1.00\left(-1.50175^{\circ}\right)$ in the right eye and -1.50 to $10 / 10$ in the left eye. In both eyes' biomicroscope examination, the anterior segment was natural; in the posterior segment, the temporal of the bilateral optic disc was pale, and the macula, retina and vitreous were within normal limits.
Table 1 Quality of life result of the case

\begin{tabular}{lll}
\hline SF-36 & $\begin{array}{l}\text { Pre-telerehabilitation } \\
\text { score }\end{array}$ & $\begin{array}{l}\text { Post-telerehabilitation } \\
\text { score }\end{array}$ \\
\hline Physical functioning & 95 & 95 \\
Role limitations due to physical health problems & 0 & 100 \\
$\begin{array}{l}\text { Role limitations due to personal or emotional } \\
\text { problems }\end{array}$ & 0 & 100 \\
Energy/fatigue & 65 & 80 \\
Emotional well-being & 60 & 88 \\
Social functioning & 37.5 & 87.5 \\
Bodily pain & 67.5 & 100 \\
General health perceptions & 70 & 80 \\
\hline
\end{tabular}


White-white perimetry and SITA-Standard central 30-2 threshold visual field test (Humphrey FieldAnalyser II -750, Zeiss-Humprey, Inc., Dublin, CA) were applied to the patient. Accordingly, peripheral scotoma, more prominent in the nasal area of the right eye visual field, and a peripheral scotoma in the form of a thin concentric narrowing were observed in the left eye. Optical coherent tomography (Cirrus ${ }^{\mathrm{TM}} \mathrm{HD}-\mathrm{OCT}$, Carl ZeissMeditec, Inc) showed that the measured mean retinal nerve fiber layer thickness was thinner than the normal population (right eye RNFL $77 \mu \mathrm{m}$, left eye $76 \mu \mathrm{m}$ ) and this thinning was more pronounced, especially in the temporal parts. Right eye vertical cup/disc ratio 0.68 ; the left eye vertical cup/disc ratio was measured as 0.73 .

When the patient came for control after 4 months of physical therapy, he stated that his double vision complaints decreased and his eyes could move more easily, especially to the right. When eye movements were evaluated, outward gaze restriction in the right eye was -2 degrees as before; in the left eye, it was determined that the eye crossed the midline more easily towards the medial and the eye movement limitation was at the level -3 (it was -4 before) towards the medial. The patient's slightly right-tilted head position was maintained. It was found that there were no change in visual acuity, anterior and posterior segment examinations, and OCT examination.

In the visual field test taken after physical therapy, it was observed that the visual field defect, which was more prominent in the right eye nasal, slightly decreased, and the visual field was enlarged (Fig. 1). It was determined that there was no obvious pathology in the visual field of the left eye. It has been demonstrated that double vision in the right gaze continues, but decreased significantly.

\section{Discussion}

MS, which can cause many eyes and vision-related diseases, can significantly reduce the quality of life of people. The most common symptoms are low vision, decreased contrast sensitivity, discomfort in binocular vision, visual field abnormalities, decreased color vision, blurred vision and double vision [7-10]. In our study, we demonstrated that the diplopia complaint in a patient with MS can be reduced by rehabilitation and that the rehabilitation approach is given via telerehabilitation provides successful results.

In a three-arm parallel randomized controlled study effects of Cawthorne-Cooksey and Frenkel exercises on balance in patients with MS were evaluated. Cawthorne-Cooksey or Frenkel exercises were applied to the intervention group for 12 weeks. The control group was taken into routine care only. As a result, it has been shown that the Cawthorne-Cooksey exercise program is more effective in improving balance in patients with MS compared to Frenkel and control groups [11].

Taking individuals with chronic diseases to face-to-face rehabilitation at the clinic due to the COVID-19 pandemic process carries a risk. Therefore, it was decided to rehabilitate the diplopia complaint of the MS patient via the telerehabilitation method. The participant reported that telerehabilitation is particularly useful for efficient use of time and is the most reliable method in the COVID-19 pandemic process. He reported that although the exercises were applied online, they were beneficial, but he sometimes had difficulties due to visual and sound problems of the internet connection. He also stated that he wanted to participate in any rehabilitation through telerehabilitation again.

The physiotherapist, who applied the telerehabilitation stated that he did not experience any problems other than minor connection problems, rarely not getting the exact feedback he wanted from the patient, and also difficulties in seeking help from the patient's relatives during the exercises.

It has been found that Cawthorne-Cooksey exercises improve the quality of life and reduce the complaints of diplopia in MS patients. In addition, the patient verbally stated that his balance increased after Cawthorne-Cooksey exercises. As a result, Cawthorne-Cooksey exercises are a rehabilitation method that provides positive results in the treatment of diplopia and it is recommended to apply this method via telerehabilitation.
Fig. 1 According to the results of the visual field tests of the patient, it is observed that the nasal peripheral vision is enlarged after physical therapy and rehabilitation in the visual field of the right eye. a Visual field test results in pre-telerehabilitation. b Visual field test results in posttelerehabilitation
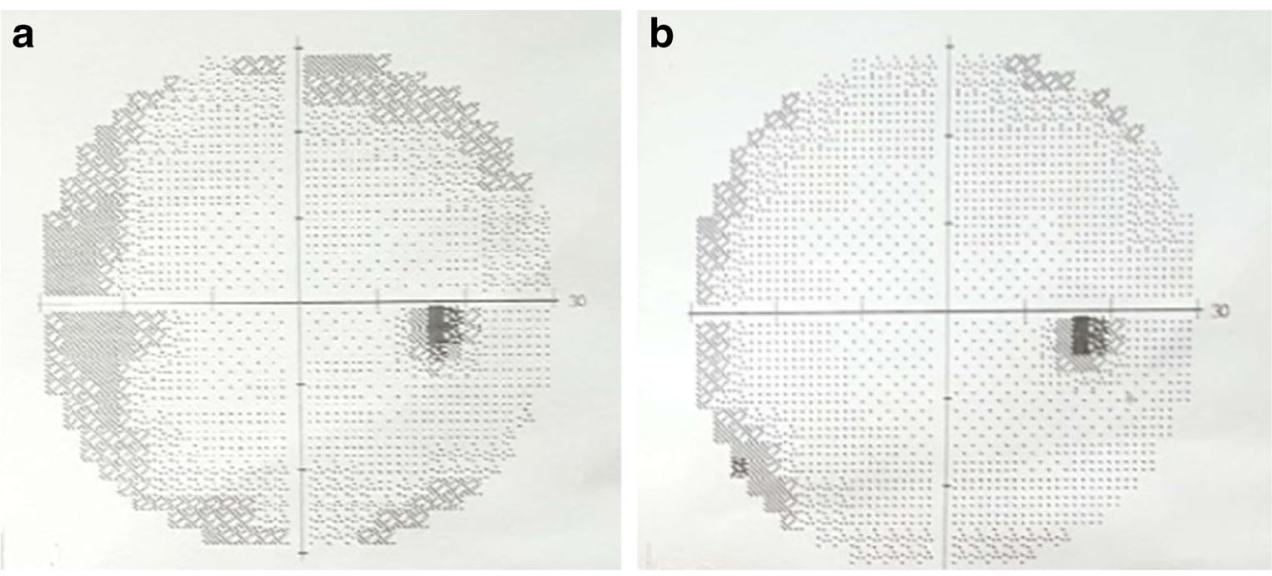
Abbreviations $M S$, Multiple sclerosis; $T R$, Telerehabilitation

Funding None.

\section{Declarations}

Conflict of interest There is no conflict of interest between authors.

Informed consent Informed consent was obtained from the patient.

Ethical approval Ethical approval was obtained from Hatay Mustafa Kemal University Ethical Council.

\section{References}

1. Akıncı B, Zenginler Y (2015) Tele-rehabilitation. Turkiye Klinikleri J Physiother Rehabil-Special Topics 1(1):14-21

2. Dogru Huzmeli E, Duman T, Yıldırım H (2017) Efficacy of telerehabilitation in patients with stroke in Turkey: A Pilot Study. Turk J Neurol 23:21-25

3. Zheng H, Black ND, Harris ND (2005) Position-sensing technologies for movement analysis in stroke rehabilitation. Med Biol Eng Comput 43(4):413-420. https://doi.org/10.1007/BF02344720

4. Balcer LJ, Miller DH, Reingold SC, Cohen JA (2015) Vision and vision-related outcome measures in multiple sclerosis. Brain $138(\mathrm{Pt}$ 1):11-27. https://doi.org/10.1093/brain/awu335
5. Hashimova Z (2018) Effectiveness of exercise treatment with visual alert in chronic unilateral vestibular hypofunction. Istanbul University Medicine Faculty Physical Therapy and Rehabilitation Department, Master Thesis, Istanbul

6. Koçyiğit H, Aydemir Ö, Ölmez N et al (1999) SF-36'nın Türkçe için güvenilirliği ve geçerliliği. İlaç ve Tedavi Dergisi 12:102-106

7. Holden MK (2005) Virtual environments for motor rehabilitation: review. Cyber Psychol Behav 8:187-211 discussion 212-9

8. van Egmond MA, van der Schaaf M, Vredeveld T, VollenbroekHutten MMR, van Berge Henegouwen MI, Klinkenbijl JHG, Engelbert RHH (2018) Effectiveness of physiotherapy with telerehabilitation in surgical patients: a systematic review and meta-analysis. Physiotherapy. 104(3):277-298. https://doi.org/10. 1016/j.physio.2018.04.004

9. Damhus C, Emme C, Hansen H (2018) Barriers and enablers of COPD telerehabilitation - a frontline staff perspective. Int J Chron Obstruct Pulmon Dis 13:2473-2482

10. Sarfo F, Ulasavets U, Opare-Sem OK, Ovbiagele B (2018) Telerehabilitation after stroke: an updated systematic review of the literature. J Stroke Cerebrovasc Dis 27(9):2306-2318

11. Afrasiabifar A, Karami F, Najafi Doulatabad S (2018) Comparing the effect of Cawthorne-Cooksey and Frenkel exercises on balance in patients with multiple sclerosis: a randomized controlled trial. Clin Rehabil 32(1):57-65. https://doi.org/10.1177/ 0269215517714592

Publisher's note Springer Nature remains neutral with regard to jurisdictional claims in published maps and institutional affiliations. 